\title{
Sequential and temporal characteristics of self and socially regulated learning
}

\author{
Inge Molenaar • Sanna Järvelä
}

Received: 10 February 2014 / Accepted: 10 February 2014 /

Published online: 5 March 2014

(C) Springer Science+Business Media New York 2014

\section{Introduction}

The conceptualization of self and socially regulated learning (S-SRL) has recently received much attention (Greene and Azevedo 2010; Järvelä and Hadwin 2013). For example, Hadwin et al. (2011) place the term social as a central for regulated learning. As such, self-regulated learning can become socially regulated learning where learner's regulatory activities are supported or constrained with others (co-regulation) or when individuals negotiate shared task perceptions, goals and strategies (socially shared regulation) (Hadwin et al. 2011; Iiskala et al. 2011; Molenaar et al. 2010; Volet et al. 2009). Moreover, most researchers agree that self and socially regulated learning are dependent of the learning situation and besides individual characteristics, the role of the learning context, task type and support should be taken into account (Boekaerts and Niemivirta 2000; Volet and Järvelä 2001). Consequently, the emphasis has been placed on capturing S-SRL as an evolving process in a learning context (Volet and Vauras 2013). Researchers have started to view S-SRL as a series of events, which can be perceived as a process that unfolds over time in a certain order (Azevedo et al. 2010; Schoor and Bannert 2012; Schraw 2010; Reimann 2009; Winne 2010).

This raises new questions with regard to the characteristics of the S-SRL process and its dynamic interplay with student and context characteristics. Addressing these issues demands for in-depth analysis of the learning process to understand how S-SRL mediates the relationship between students' characteristics, contexts' characteristics and performance (Greene and Azevedo 2010). However until now the S-SRL process is mostly studied by counting the number of self-regulating learning strategies students perform (Azevedo et al. 2008; Molenaar et al. 2010). In many cases the S-SRL process is taken as one holistic unit and its sequential and temporal characteristics are therefore largely ignored (Kapur 2011; Reimann 2009; Schmitz 2006; Schoor and Bannert 2012). The sequential characteristics of S-SRL consider which actions typically follow each other, and temporal characteristics of those actions tells about when those actions are taken and how they influence each other over time (Reimann

I. Molenaar ( $\square)$

Radboud University Nijmegen, Montessorilaan 3, Nijmegen, The Netherlands

e-mail: i.molenaar@pwo.ru.nl

S. Järvelä

University of Oulu, Oulu, Finland 
2009). In this light, several researchers have expressed the need to explore time and order in SSRL processes (Greene and Azevedo 2010; Molenaar et al. 2011; Schmitz 2006; Winne 2010).

This special issue illustrates how empirical studies of sequential and temporal characteristics of S-SRL provide new insights that enhance our theoretical and practical understanding of S-SRL. The contributors of this special issue are leading international researchers who articulate different emerging perspectives on time and order in S-SRL. In this special issue, all articles analyze sequential and/or temporal characteristics of S-SRL. Focussing on different subjects (pre-school students to university students) and performing these analyses in the light of different research questions, each contribution has its own individual theoretical background. In all, these studies show that not only the prevalence of S-SRL actions, but also their sequential and temporal characteristics, are important.

Apart from their individual contributions to our understanding of time and order in S-SRL process, all contributions also differ in respect to the methods used to analyze these sequential and temporal characteristics. Five empirical contributions show how emerging methods (statistical discourse analysis, t-pattern analysis, fuzzy data mining, Markov modeling and trace data analysis) are used to explore time and order in the S-SRL process. These methods originate from different methodological traditions and each of the papers specifies a methodological approach in detail. The contributions of these papers are unique and representative for our current understanding of the temporal characteristics of S-SRL. The commentaries of Roger Azevedo and Phil Winne will discuss the commonalities and differences among findings and methods. As such this special issue constitutes a first step towards an understanding the sequential and temporal characteristics of S-SRL and formulating an approach to investigate time and order in S-SRL.

\section{The sequential and temporal characteristics of S-SRL}

Self and socially regulated learning theory (S-SRL) defines learning as a goal oriented process, that assumes students make conscious choices working toward their self or socially defined learning goals (Boekaerts 1999; Hadwin and Järvelä 2011; Zimmerman 2002). Self-regulating learners use cognitive activities (read, process, elaborate) to study a topic and effectively control and monitor their learning with metacognitive activities (orientate, plan, monitor and evaluate their actions) and motivate themselves to an appropriate level of engagement (Azevedo et al. 2008; Winne and Hadwin 2010; Zimmerman 2002). Hence S-SRL involves a complex interplay of cognitive, metacognitive, and motivational regulatory components. Although theories of S-SRL vary in their concentration these components, they do share important aspects assumptions about the sequential and temporal characteristics of S-SRL (Hadwin 2011).

The notion of learners' agency is well grounded in S-SRL theory. It is assumed that learners' learning goals steer their learning, which entails the assumption that learners project these learning goals over time (Hadwin 2011). Moreover, there seems to be consensus about three important phases in the process of self-regulation (Zimmerman 2002; Winne \& Hadwin 1998). Namely the preparation phase, the execution phase and the reflection phase. In the preparation phase students orientate on the task, activate existing knowledge and set their learning goals. These goals guide the execution phase in which students perform the learning task. Finally students reflect on their approach toward the task in order to optimize the process in the future.

The idea that S-SRL unfolds in the three main phases suggests a cyclical relation among the components. Clearly et al. (2012) uses the term "sequential phases of regulation" to describe 
the cyclical nature of regulated learning. This sequential notion is used in S-SRL theories to explain how students move through different phases of S-SRL to guide their learning. However, there is no view of strict order in this cyclical notion (Greene and Azevedo 2010). Even though the S-SRL process is considered cyclical, learners can jump between different SSRL actions inducing recursive sequential patterns within the cycle. This raises questions concerning the dynamics of this cyclical S-SRL process, the sequential patterns within it as well as the development of the cycle over time. Moreover, there are some differences with regard to the time window that the S-SRL cycle should be projected on. For example, Winne and Hadwin (2013) support a micro level perspective on S-SRL specifying the COPE model as cycle that re-occurs within one study session, whereas the cycle from Zimmerman (2002) is a more macro level cycle encapsulating one learning task possibly extending over multiple study sessions.

Thus assumptions about the sequential and temporal characteristics of S-SRL are grounded in our theoretical models. The sequential characteristics of S-SRL entail transitions from one action and/or phase to another (e.g. transition from preparation phase to the execution phase). The temporal characteristics focus on when S-SRL actions and sequences are taking place and how they act over time. Existing research about the temporal characteristics of S-SRL can be viewed in the light of two distinct views on temporality, namely analyzing events in a continuous flow or focusing on relative arrangements among multiple events (Molenaar and Wise, in prep).

The first view on temporality views events in a continuous flow. Here the focus lays on the individual temporal characteristics of S-SRL actions and how these characteristics act within the learning process as a whole. This type of research analyzes the positioning of S-SRL actions, their duration and/or the rate at which they occur during learning. For example, Johnson et al. (2011) showed that planning activities occur more frequently later in the learning task. In this special issue Kuvalja and colleagues (2014) indicate that there are no differences in the rate of self-regulation behaviors between typically developing children and children with specific language disorder.

The second view on temporality analyzes relative arrangement among multiple events, which embodies a different perspective on time. Here, the focus does not lay on the individual temporal characteristics of S-SRL actions, but on the organization of these actions addressing the relative arrangement of multiple S-SRL actions over time. For example, in this special issue Bannert and colleagues (2014) show that the SRL process of successful students is more similar to our theoretical model of SRL compared to the SRL process of unsuccessful students. Investigating the development of sequential characteristics of S-SRL also falls under this view on temporality. For example, Molenaar and Chiu (2014) show that there is remarkable stability in sequential relations between socially regulated learning actions and cognitive actions. Finally, one can study transformations of sequences looking at their duration and fluctuations over time. For example, Kuvalja and colleagues (2014) show that children with specific language disorder show self-directed speech and self-regulation behavior in sequences that are longer and more complex than typically developing children.

Yet, despite these theoretical assumptions of sequential and temporal characteristics, comprehensive research collecting empirical findings to support these theories is scarce and there have been few validations in real learning settings (Bannert and Mengelkamp 2013; Biswas et al. 2010; Greene and Azevedo 2007; Greene and Azevedo 2010; Schoor and Bannert 2012; Winne and Nesbit 1995). Therefore, the objective of this special issue is to explore the time and order in S-SRL processes to further enhance our theoretical understanding of the construct and how its' dynamic characteristics influence learning. These analyses can be done focussing on the individual sequential and temporal characteristics of S-SRL actions and/or by 
investigating the temporality of sequential characteristics. This information is essential to understand contextual features of regulated learning in individual and social settings. For most contributors, this research is eventually aimed at developing meaningful interventions to support learners to apply these processes more effectively.

\section{The contribution of this special issue for understanding sequential and temporal characteristics of self and socially regulated learning}

The five papers in this special issue show how different researchers in the field of S-SRL analysed time and order in S-SRL processes. What is specific, is that research questions drove inquiries embedded in different theoretical backgrounds incorporating various perspectives on time. What is common, is that all papers applied different methodologies to explore the temporal characteristics of S-SRL. Below, we will discuss each of the papers shortly in the light of both their theoretical contribution and their methodological approach.

The first paper by Kuvalja and colleagues (2014) focused on the mediating role of selfdirected speech in emerging self-regulatory behavior in young children. They investigated real-time temporal interactions between self-directed speech and self-regulatory behavior in children with specific language disorder in comparison with typically developing children. Often this comparative research is performed using correlation methods which are unable to capture the temporal characteristics the authors are interested in. They combine the two views of temporality looking at the prevalence and the co-occurrence of self-directed speech and selfregulatory behaviour.

In Kuvalja et al. (2014) self-directed speech and self-regulatory behaviours were observed and coded from childerns' verbal and non-verbal behaviour. The authors used frequency analysis to assess the prevalence of the behaviours, sequential lag analysis to determine adjacent sequences of behaviours and temporal ( $\mathrm{t}$ )-pattern analysis to search for recurring non-adjacent patterns. Frequency analysis showed no differences in the rate of self-directed speech or self-regulatory behaviors between the two groups. Nor were differences found in adjacent order between the self-directed speech an self-regulatory behaviour between the two groups with sequential lag analysis. T-patterns did show a difference between the two groups: temporal patterns of self-directed speech and self-regulatory behaviour of children with specific language disorder were more in number, more complex and typically included selfdirected speech utterances.

In this study, the researchers elegantly showed the importance of t-pattern analysis in its ability to detect extended temporal structures in the data that otherwise would have remained undetectable. These differences between the two groups could not be revealed using either frequency analysis or sequential lag analysis. The authors indicated that t-pattern analysis is an useful method for studying development as it unfolds over time. They do conclude that more insights in the use of this method and the setting of parameters is needed for the field to progress.

Malmberg, Järvelä and Kirschner (2014) investigated the temporal characteristics of students' strategies use during learning making a distinction between students' behaviour in ill and well-structured tasks. Their research is grounded in the assumption that the learning task influences how students self-regulate their learning. Specifically, Malmberg et al. (2014) were interested if, how and when students' apply strategies aligned with the task type and on/offtrack task performance. This study aimed to show that trace based methods are an appropriate solution for measurement problems of self-regulated learning where either students over- 
estimate their skills in self-reports or thinking aloud procedures impediment their learning performance.

Based on log file events from the computer based learning environment gStudy, the students' strategic actions were determined. Transition matrix's defined the sequences among these strategic actions. Sequences that occurred more than 3 times were considered as learning patterns. As such the authors also combined the two perspectives on temporality analyzing the prevalence of strategies and learning patterns. They showed that although there was no difference in the amount of on /off-track task solutions between well- and ill-structured tasks, the learning patterns in ill-structured tasks seem less strategic. These patterns were oriented more towards understanding the task by explaining and questing, whereas in well-structured tasks these patterns were more directed at information selection. Additionally, there were indications of intra-individual differences in the use of learning patterns over time. On-track students applied learning strategies in later phases compared to off-track students.

Malmberg et al. (2014) indicated that investigating intra-individual differences in students' strategies can contribute to our understanding how students self-regulate their learning. This requires the application of mixed methods designs and process-oriented approaches with a focus on sequential and temporal characteristics of strategy use. These analyses will elicit insights into how students allocate their strategic actions and how that relates to their task understanding.

Molenaar and Chiu (2014) focused on the relative arrangements of multiple events perspective on temporality analyzing how different learning activities alternate during collaborative learning and the role of scaffolding of self-regulated learning there on. The rational for this study were their earlier findings that cognitive, metacognitive and relational activities contribute significantly to learning, yet the micro-level relations among these activities and how they support effective collaborative learning were unknown. Therefore, Molenaar and Chiu (2014) explored how sequences of students' cognitive, metacognitive and relational activities affect the likelihoods of subsequent low vs. high cognitive activities during collaborative learning. Moreover, they investigated whether these arrangements remained stable over the instructional time analyzed.

In their study, three methods were applied; namely content analysis to determine different learning activities in the groups discourse; statistical discourse analysis to model the sequential relations among these activities and, finally, discourse analysis to show representative excerpts to explicate the relationships among these learning activities. Analysing the behaviour of low and high cognition during the instructional unit $(6 \mathrm{~h})$ indicated that there were intra-group differences in the use of low and high cognition over time, but no stable time periods could be depicted across groups.

Results indicated that there were remarkably long lasting (6 lags) positive effects of both low and high cognition on itself. Among metacognitive activities, planning, monitoring and evaluation immediately aided low cognition, whereas planning and evaluation supported high cognition with a lag of 2 or 3 events. Orientation reduced the likelihood of high cognition. A reoccurring sequence suggested the facilitating role of planning in transitions between low and high cognition: namely planning induced low cognition which then sparked high cognition. Finally, scaffolding was related to high cognition but it did not alter the sequences found among the learning activities. This study contributed to our micro level understanding of how regulation is embedded in collaborative learning. The stability found among learning activities encourages further exploration of temporal characteristics of socially regulation building towards micro interaction model of socially regulated learning.

Bannert, Reimann and Sonnenberg (2014) researched students' spontaneous use of SRL strategies focussing especially on the temporal order among these strategies. Based on the assumption that there are intra-individual differences between successful and unsuccessful 
students, they compared the sequential relations among self-regulated learning strategies between these two groups. The main view on temporality in this study was on the arrangement of multiple events. The researchers used content analysis of students' think aloud protocols to determine SRL actions and process mining to model self-regulated learning processes of students. According to the authors, process mining is an appropriate analysis method to provide insight into the temporal characteristics of students' self-regulated learning. Process mining does not consider time as a continuous variable, but it can identify, confirm and extend process models based on event based data.

Bannert et al. (2014) results showed that the process models of successful and unsuccessful students differed both in the amount of self-regulated learning events they performed as well as in the relations between these events. Yet, in order to compare the process models found by the process mining algorithms with our theoretical models of self-regulated learning, the authors needed to aggregate the coded data into new categories clustering different events. Consequently, the results indicated that the self-regulated learning process model of successful students came closer the expectations of self-regulated learning theory than the process model of unsuccessful students. These students analysed, monitored, read, processed and evaluated, whereas, unsuccessful students analysed, read, repeated, processed and monitored but did not evaluate their learning.

This study contributed to our understanding of how regulatory activities unfold over time and contributed to empirical evidence of the sequential characteristics and the cyclical process in self-regulation theory. As next steps Bannert et al. (2014) suggested that the field can benefit from data mining methods, but that we need more knowledge to use these methods appropriately. Moreover, data mining efforts should be guided by a theoretical framework that matches with the level of the data coded. Currently, we lack a micro-level model of SRL to guide these efforts at the appropriate granularity level.

Kinnebrew and colleagues (2014) started from the need for adaptive scaffolding to support students' self-regulated learning in their computer based learning environment called Betty's Brain. They envision a system that interprets students' actions during learning to adaptively scaffold students' progress when they are learning. However, currently it is difficult to accurately infer students' use of strategies based on the traces they leave in computer based learning environments. The authors used exploratory data mining techniques to track and identify students' use of cognitive and metacognitive strategies. Especially the use of their cognitive/metacognitive task model helped to map the logfile traces on students' strategy use. The methods applied are hierarchical clustering method to group patterns with similar trends in occurrence over time and sequential pattern mining to find patterns of observed behaviors that are common among groups of students. Finally, heatmaps were used as a visualisation method to compare how scaffolding conditions differed in their strategies use over time.

In this study they compared two experimental conditions receiving different kind of scaffolds with a control condition. The experimental groups received different forms of scaffolding, knowledge construction support or solution evaluation strategies. Although the three conditions do not differ in learning gains, exploratory data mining methods did show differences in strategy use between the three conditions. Specifically, both groups receiving scaffolds showed the strategy use induced by the scaffolding within the time window of the scaffolding. The heatmaps clearly indicated the differences in strategy use between the conditions.

As a next step, Kinnebrew et al. (2014) study indicated a need to refine their methods to better characterize and understand strategies use during learning. Especially, the integration of more detailed contextual information to better understand students learning behaviour is indicated as a promising direction. Moreover, they indicated the need for more microgenetic 
analysis of students' metacognitive strategies during learning in Betty's Brain to understand and validate the behavior patterns found through data mining.

To summarise, Table 1 outlines the main aspects of the contributions in this special issue. All contributions looked at sequential characteristics of S-SRL and most analyzed the temporal characteristics as relative arrangements of multiple events, which is closely connected to the theoretical notion of self and socially regulated learning as a series of SRL events that act dynamically over time and contexts. The first two papers (Kuvalja et al. 2014 and Malmberg et al. 2014) also incorporate the analysis of individual temporal characteristics of events and show how both perspectives on temporality provide different insights into sequential and temporal characteristics of self-regulatory behaviour and strategy use. The research questions addressed are mostly exploratory and comparative addressing differences in sequential and temporal characteristics of self-regulated learning between different groups. The studies in this special issue did not explore the connection between these characteristics of self-regulated learning and students' learning gains. Although in some contributions (Kinnebrew et al. 2014; Malmberg et al. 2014), when no differences in learning gains were found between the groups compared, there were substantial differences between the groups in temporal and/or sequential characteristics of strategy use.

All studies have defined the time window investigated based on the instructional unit, except for Molenaar and Chiu who additional looked at the homogeneity of the use of low and high cognition over time to further segment time and Kinnebrew and colleagues who divided the instruction unit in 5 additional time windows. Although data used in the analysis vary from observation data (Kuvalja et al.), log file data (Malmberg et al. 2014; Kinnebrew et al. 2014), think aloud data (Bannert et al. 2014), discourse data (Molenaar and Chiu 2014), all contributors used online measurement of S-SRL and view the construct as a series an events. The methods used come from a range of methodological backgrounds from such as statistics (Molenaar and Chiu 2014), data mining methods (Bannert et al. 2014; Kinnebrew et al. 2014; Malmberg et al. 2014) and system dynamics (Kuvalja et al. 2014). Most authors pointed out the need of a more advanced understanding of the methods used. For example Bannert et al. 2014 and Kuvalja et al (2014). indicated the importance of making more guidelines for setting parameters and significance levels when applying algorithms.

From these studies, we can see that the connection between studies into temporal characteristics and self-regulated learning theory is challenging. These papers analysed self-regulated learning at a micro level and a number of authors (Bannert et al. 2014; Kinnebrew et al. 2014 and Molenaar \& Chiu (2014) signposted the need for more micro level theory to further articulate our understanding of the temporal characteristics of self and socially regulated learning. Moreover, intra-individual differences in temporal use of self-regulated learning need future attention (Bannert et al. 2014; Malmberg et al. 2014 and Molenaar \& Chiu 2014).

This is the first time that a special issue is specifically focused on sequential and temporal characteristics of S-SRL. The authors reported new methodological techniques to analyze time and order in S-SRL processes and these contributions show evidence that the use of selfregulated learning strategies is dynamic over time, contexts and student characteristics, which provides empirical support for the conceptualisation of self and socially regulated learning as event-based constructs. Stability over time, context and students characteristics on the other hand would have pointed towards trait-related behavior (Schmitz 2006). To conclude, this special issue shows the importance of research into the sequential and temporal characteristics of self and socially regulated learning. These contributions also embody an articulation of the need to enhance our understanding of the temporal characteristics in general in the field of learning and instruction. 


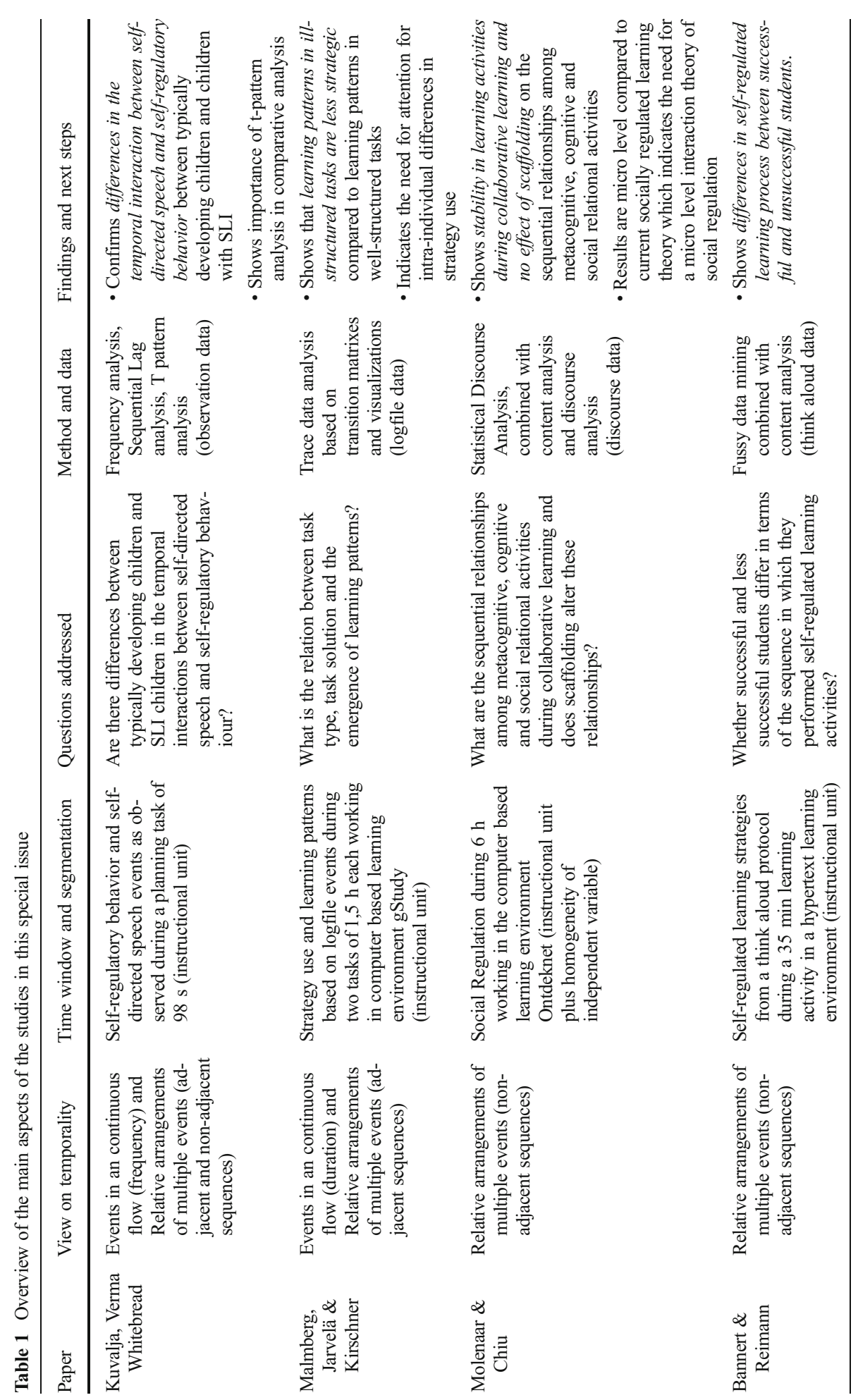




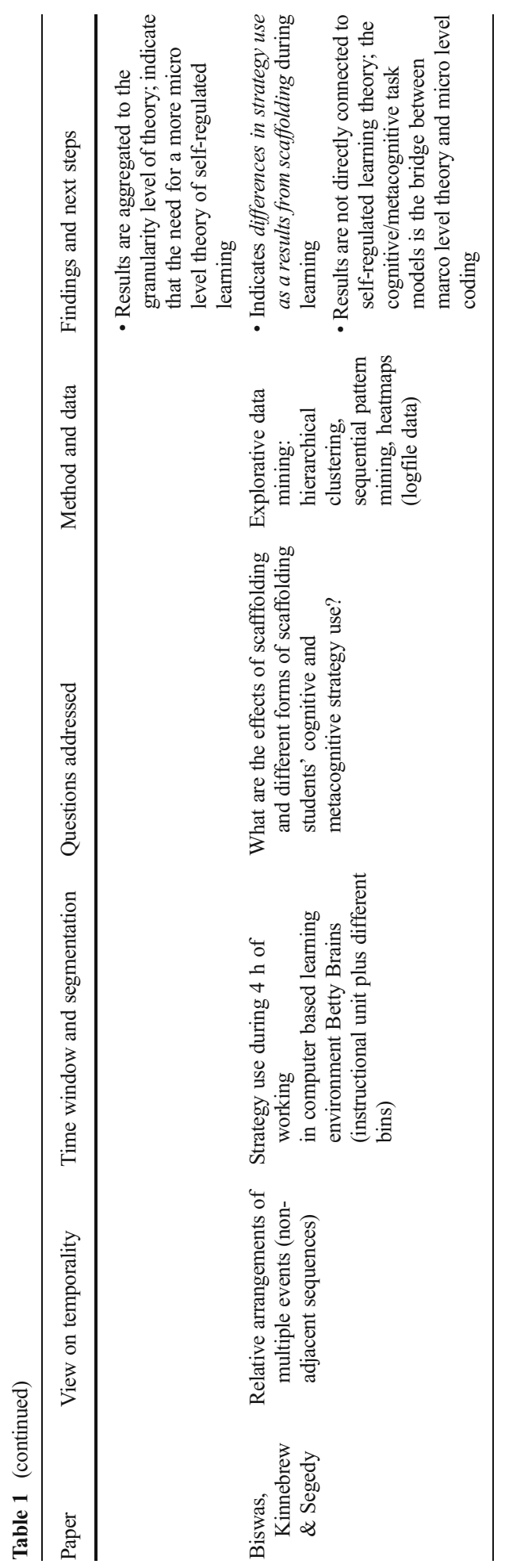




\section{References}

Azevedo, R., Moos, D. C., Greene, J. A., Winters, F. I., \& Cromley, J. G. (2008). Why is externally-facilitated regulated learning more effective than self-regulated learning with hypermedia? Educational Technology Research and Development, 56(1), 45-72.

Azevedo, R., Moos, C. D., Johnson, A. M., \& Chauncey, A. D. (2010). Measuring cognitive and metacognitive regulatory processes during hypermedia learning: issues and challenges. Educational Psychologist, 45, 210223.

Bannert, M., \& Mengelkamp, C. (2013). Scaffolding hypermedia learning through metacognitive prompts. In International Handbook of Metacognition and Learning Technologies (pp. 171-186). Springer New York.

Bannert, M., Reimann, P., Sonnenberg, C. (2014). Process mining techniques for analysing patterns and strategies in students' self-regulated learning. Metacognition and learning, 9. doi:10.1007/s11409-0139107-6

Biswas, G., Jeong, H., Kinnebrew, J., Sulcer, B., \& Roscoe, R. (2010). Measuring self-regulated learning skills through social interactions in a teachable agent environment. Research and Practice in TechnologyEnhanced Learning (RPTEL), 5(2), 123-152.

Boekaerts, M. (Ed.). (1999). Self-regulated learning. International Journal of Educational Research, 31(6).

Boekaerts, M., \& Niemivirta, M. (2000). Self-regulation in learning: Finding a balance between learning- and ego-protective goals. In M. Boekaerts, P. R. Pintrich, \& M. Zeidner (Eds.), Handbook of self-regulation (pp. 417-450). San Diego: Academic.

Cleary, T. J., Callan, G., \& Zimmerman, B. J. (2012). Assessing self-regulation as a cyclical,context-specific phenomenon: Overview and analysis of SRL microanalytic protocols. Education Research International. doi: $10.1155 / 2012 / 428639$.

Greene, J. A., \& Azevedo, R. (2007). A theoretical review of Winne and Hadwin's model of self-regulated learning: new perspectives and directions. Review of Educational Research, 77(3), 334-372.

Greene, J. A., \& Azevedo, R. (2010). The measurement of learners'self-regulated cognitive and metacognitive processes while using computer-based learning enviroments. Educational Psychologist, 45, 203-209.

Hadwin, A.F. (2011). Self-regulated learning. In Good, T. L. (Ed). 21st Century Education; A Reference Handbook: (Vols. 1-2). Thousand Oaks, CA: SAGE Publications.

Hadwin, A. F., \& Järvelä, S. (2011). Introduction to a special issue on social aspects of self-regulated learning: where social and self meet in the strategic regulation of learning. Teachers College Record, 113(2), 235-239.

Hadwin, A., Järvelä, S., \& Miller, M. (2011). Self-regulated, co-regulated, and socially shared regulation of learning. In B. Zimmerman \& D. Schunk (Eds.), Handbook of self-regulation of learning and performance (pp. 65-84). New York: Routledge.

Iiskala, T., Vauras, M., Lehtinen, E., \& Salonen, P. (2011). Socially shared metacognition of dyads of pupils in collaborative mathematical problem-solving processes. Learning and Instruction, 21(3), 379-393.

Järvelä, S., \& Hadwin, A. (2013). New frontiers: regulating learning in CSCL. Educational Psychologist, 48(1), 25-39.

Johnson, A. M., Azevedo, R., \& D’Mello, S. K. (2011). The temporal and dynamic nature of self-regulatory processes during independent and externally assisted hypermedia learning. Cognition and Instruction, 29(4), 471-504.

Kapur, M. (2011). Temporality matters: advancing a method for analyzing problem-solving processes in a computer-supported collaborative environment. International Journal of Computer-Supported Collaborative Learning (ijCSCL), 6(1), 39-56.

Kinnebrew, J.S., Segedy J.R., Biswas, G. (2014). Analyzing the temporal evolution of students' behaviors in open-ended learning environments. Metacognition and learning, 9 doi:10.1007/s11409-014-9112-4.

Kuvalja, M., Verma, M., Whitebread, D. (2014). Patterns of co-occurring non-verbal behavior and self-directed speech; a comparison of three methodological approaches. Metacognition and learning, 9. doi:10.1007/ s11409-013-9106-7.

Malmberg, J., Järvelä, S., Kirchner, P. (2014). Elementary school students’ strategic learning: does task-type matter? Metacognition and learning, 9. doi:10.1007/s11409-013-9108-5.

Molenaar, I. \& Wise, A. (in prep.). A conceptual framework for temporal analysis.

Molenaar, I \& Chiu M.M. (2014). Dissecting sequences of regulation and cognition: statistical discourse analysis of primary school children's collaborative learning. Metacognition and learning, 9. doi:10.1007/s11409013-9105-8

Molenaar, I., van Boxtel, C. A. M., \& Sleegers, P. J. C. (2010). The effects scaffolding metacognitive activities in small groups. Computers in Human Behavior, 26(6), 1727-1738.

Molenaar, I., Chiu, M. M., Sleegers, P. J. C., \& van Boxtel, C. A. M. (2011). Scaffolding of small groups' metacognitive activities with an Avatar. International Journal of Computer Supported Collaborative Learning, 6(4), 601-624.

Reimann, P. (2009). Time is precious: variable- and event-centred approaches to process analysis in CSCL research. International Journal of Computer-supported Collaborative Learning, 3, 239-257. 
Schmitz, B. (2006). Advantages of studying processes in educational research. Learning and Instruction., 16, $433-449$.

Schoor, C., \& Bannert, M. (2012). Exploring regulatory processes during a computer-supported collaborative learning task using process mining. Computers in Human Behavior, 28(4), 1321-1331.

Schraw, G. (2010). Measrunign self-regulation in computer-based learning enviroments. Educational Psychologist, 45, 258-266.

Volet, S., \& Järvelä, S. (Eds.). (2001). Motivation in learning contexts: Theoretical advances and methodological implications. London: Pergamon/Elsevier.

Volet, S., \& Vauras, M. (2013). Interpersonal regulation of learning and motivation: Methodological advances. Abingdon: Routledge, part of the Taylor \& Francis Group.

Volet, S., Summers, M., \& Thurman, J. (2009). High-level co-regulation in collaborative learning: how does it emerge and how is it sustained? Learning and Instruction, 19, 128-143.

Winne, P. H. (2010). Improving measurements of self-regulated learning. Educational Psychologist, 45, 267276.

Winne, P. H., \& Hadwin, A. F. (1998). Studying as self-regulated learning. In D. J. Hacker, J. Dunlosky, \& A. C. Graesser (Eds.), Metacognition in educational theory and practice (pp. 277-304). Mahwah, NJ: Lawrence Erlbaum Associates.

Winne, P. H., \& Hadwin, A. F. (2010). Self-regulated learning and socio-cognitive theory. International encyclopedia of education, 5, 503-508.

Winne, P. H., \& Hadwin, A. F. (2013). Study: Tracing and supporting self-regulated learning in the Internet. In International handbook of metacognition and learning technologies (pp. 293-308). Springer New York.

Winne, P. H., \& Nesbit, J. C. (1995). Graph theoretic techniques for examining patterns and strategies in students' studying: An application of LogMill. Paper presented at the American Educational Research Association, April 1995.

Zimmerman, B. J. (2002). Becoming a self-regulated learner: an overview. Theory into Practice, 42(2), 64-70. 